\title{
Structural basis of the nucleosome transition during RNA polymerase II passage
}

\author{
Tomoya Kujirai1 $^{1,2 *}$, Haruhiko Ehara ${ }^{2 *}$, Yuka Fujino ${ }^{1,3}$, Mikako Shirouzu${ }^{2}$, Shun-ichi Sekine ${ }^{2}+$, \\ Hitoshi Kurumizaka ${ }^{1,2,3} \uparrow$ \\ ${ }^{1}$ Laboratory of Chromatin Structure and Function, Institute for Quantitative Biosciences, The University of Tokyo, 1-1-1 Yayoi, Bunkyo-ku, Tokyo 113-0032, Japan. ${ }^{2}$ RIKEN \\ Center for Biosystems Dynamics Research, 1-7-22 Suehiro-cho, Tsurumi-ku, Yokohama 230-0045, Japan. ${ }^{3}$ Graduate School of Advanced Science and Engineering, Waseda \\ University, 2-2 Wakamatsu-cho, Shinjuku-ku, Tokyo 162-8480, Japan. \\ *These authors contributed equally to this work. \\ †Corresponding author. Email: kurumizaka@iam.u-tokyo.ac.jp (H.K.); shunichi.sekine@riken.jp (S.S.)
}

Genomic DNA forms chromatin, in which the nucleosome is the repeating unit. The mechanism by which RNA polymerase II (RNAPII) transcribes the nucleosomal DNA remains unclear. Here we report the cryoelectron microscopy structures of RNAPII-nucleosome complexes, in which RNAPII pauses at the superhelical locations, SHL(-6), SHL(-5), SHL(-2), and SHL(-1), of the nucleosome. RNAPII pauses at the major histone-DNA contact sites, and the nucleosome interactions with the RNAPII subunits stabilize the pause. These structures reveal snapshots of nucleosomal transcription, where RNAPII gradually tears DNA from the histone surface, while preserving the histone octamer. Interestingly, the nucleosomes in the SHL(-1) complexes are bound to a "foreign" DNA segment, which might explain the histone transfer mechanism. These results provide the foundations for understanding chromatin transcription and epigenetic regulation.

In eukaryotes, genomic DNA is packaged as chromatin, in which the nucleosome is the basic unit (1). In the nucleosome, a 145 - to 147 -base pair DNA segment is stably wrapped around a histone octamer, composed of two each of histone $\mathrm{H} 2 \mathrm{~A}-\mathrm{H} 2 \mathrm{~B}$ and $\mathrm{H} 3-\mathrm{H} 4$ heterodimers (2). Since the nucleosome has an extremely stable architecture, it strongly affects genomic DNA functions, such as transcription (3-5). RNA polymerase II (RNAPII), a multi-subunit molecular machine (6), transcribes protein-coding genes, but the mechanism of nucleosome transcription remains obscure.

To address this problem, we performed cryo-electron microscopy (cryo-EM) single-particle analyses of transcribing RNAPII-nucleosome complexes. In cells, RNA elongation by RNAPII is stalled at multiple sites within a nucleosome up to the nucleosomal dyad (7). After passing through the nucleosomal dyad, RNAPII transcribes the DNA without obvious impediments (7), because the histones may be removed from the DNA ahead of the RNAPII. To avoid the histone dismantling by RNAPII, we designed a nucleosomal template with a T-less sequence region, by which transcription elongation is stalled at the SHL(-1) position, a natural RNAPII pausing site located about 10 base-pairs upstream of the nucleosomal dyad, in the presence of 3 '-dATP (fig. S1A). Widom 601, an artificial sequence with high nucleosome positioning power, was employed for the design (8). After reconstitution of the human nucleosome with the DNA, a linker DNA containing the transcription bubble was ligated to one end of the nucleosomal DNA (fig. S1, A to D). RNA elongation was performed by RNAPII from the yeast Komagataella pastoris (fig. S1E). RNAPII predominantly paused at the entry of the nucleosome $[\mathrm{SHL}(-5)]$, reflecting the rate-limiting step of the nucleosomal transcription in vitro (fig. S1F). In contrast, RNAPII elongated RNA until the SHL(-1) position in the presence of the transcription elongation factor TFIIS (fig. S1E), which facilitates the nucleosomal transcription (fig. S1F) (9, 10).

The obtained transcribing complexes, containing RNAPII, nucleosomes, and transcribed RNAs, were partially purified by the sucrose-glutaraldehyde gradient ultracentrifugation (GraFix) method (11). The RNAs within the RNAPIInucleosome complex mixture were then analyzed by denaturing polyacrylamide gel electrophoresis (Fig. 1A). Significant pausing of RNAPII was observed near the entry [SHL(-5)] and before the dyad [SHL(-1)] of the nucleosome (Fig. 1A). These major pausing sites correspond well to the in vivo pausing sites within gene-body nucleosomes (7). In addition, minor, but clear, RNA bands corresponding to the paused RNAPII at SHL(-6) and SHL(-2) were detected (Fig. 1A).

We then collected cryo-EM images of the transcription reaction mixtures, and extensive 3D classifications delineated seven distinct states of the RNAPII-nucleosome complexes, which belong to the complexes paused around SHL(-6), SHL(5), SHL(-2), and SHL(-1) (Fig. 1, B to E; figs. S2 to S6; tables $\mathrm{S} 1$ and S2; and movie S1). These structures revealed the 
nucleosome transition during the RNAPII passage, while no major conformational change was observed in the RNAPII (see materials and methods). In these complexes, RNAPII is stalled at the major contact sites between the DNA and the core histones (Fig. 2). These sites are either the H2A-H2B contact site [SHL(-5)] or the H3-H4 contact sites [SHL(-6), SHL(2), and SHL(-1)] (Fig. 2). These strong histone-DNA interactions should be the major cause of the RNAPII pausing, and thus explain the previous observations that the histone mutations that weaken histone-DNA interactions relieve the RNAPII pausing (12). Interestingly, the nucleosome orientations relative to RNAPII are similar among these structures (fig. S7, A to E), in which the curved nucleosomal DNA fits on the RNAPII surface, interacting with the clamp head domain of the Rpb1 subunit (fig. S7F). These RNAPII-DNA interactions seem to stabilize the RNAPII stalled at each SHL.

In the RNAPII-nucleosome complexes stalled around SHL(-6) and SHL(-5), the RNAPII is located near the entrance of the nucleosome. In the SHL(-6) complex, the nucleosomal DNA is entirely wrapped around the histone octamer (Figs. $1 \mathrm{~B}$ and $2 \mathrm{~A})$. In the SHL(-5) complex, the RNAPII had advanced by approximately $20 \mathrm{bp}$, and the DNA end of the nucleosome is torn away from the $\mathrm{N}$-terminal part of histone $\mathrm{H} 3$ (Figs. $1 \mathrm{C}$ and 2B). The $\mathrm{H} 3 \mathrm{~N}$-terminal region contains many modification sites, which may affect the DNA affinity to the histone surface (13-15). Similar 20 bp detachment reportedly occurs by nucleosome remodelers, such as CHD1 and INO80 (16-18). Therefore, the histone modifications and the nucleosome remodelers may modulate the nucleosome accessibility of RNAPII at the nucleosomal entry site.

Before the dyad, RNAPII is stalled at the SHL(-2) and SHL(-1) positions of the nucleosome (Figs. 1, D and E, and 2, $\mathrm{C}$ and D). In the SHL(-2) and SHL(-1) complexes, about $50 \mathrm{bp}$ and $60 \mathrm{bp}$ DNA regions are peeled away from the histone surface by RNAPII, respectively. An H2A-H2B dimer is reportedly released from the nucleosome when a $40 \mathrm{bp}$ stretch of the nucleosomal DNA is removed (19). However, the H2AH2B dimer density is clearly observed in the SHL(-1) and SHL(-2) complexes (Fig. 3, A and B). Consistently, no nucleosome particles lacking H2A-H2B were observed during the classification processes. In the SHL(-2) complex, a density connection is observed between the Rpb2 lobe domain of RNAPII and the H2A-H2B dimer, and this interaction may retain the H2A-H2B dimer in the nucleosome (Fig. 3C).

The majority of the complexes stalled at SHL(-1) contained a $\sim 60$ bp DNA segment of unknown origin ("foreign" DNA), which is bound to the DNA-peeled region of the nucleosome (fig. S8, A to C). Foreign DNA association was also observed in the non-crosslinked sample (fig. S9), indicating that the DNA binding is not due to crosslinking. Further analyses of these complexes yielded three distinguishable classes with the foreign DNA: the SHL(-1), tilted SHL(-1), and SHL(-1)+1 complexes (fig. S8, A to C). In the SHL(-1) $)_{+1}$ complex, RNAPII proceeded by one base pair (fig. S8C), and rotated by about $36^{\circ}$ (fig. S8D and movie S2), as compared with those positions in the SHL(-1) complex. The tilted SHL(-1) complex seems to be an intermediate between the SHL(-1) and SHL(-1) $)_{+1}$ complexes (fig. S8, B and D, and movie S2). In these complexes, RNAPII is stalled due to the DNA contacts with histones H3$\mathrm{H} 4$. Interestingly, the H3K64 acetylation, which may reduce the H3-DNA contact at the SHL(-1) position (Fig. 2D), is reportedly accumulated at the transcription start sites of active genes (20). Therefore, SHL(-1) is probably another important site for transcriptional regulation coupled with histone modifications.

The foreign DNA is derived from a linker DNA region of other nucleosomes or disassembled nucleosomes. The former particles are observed in the raw cryo-EM images (fig. S10). This suggested that the DNA-peeled region (mainly H2AH2B) has a propensity to associate with DNA to regenerate nucleosome-like structures. Therefore, the foreign DNA could serve as an intermediate for histone transfer to other DNA regions in cis/trans. It has been proposed that the histone is transferred from ahead to behind the transcribing RNA polymerase via a "template looping" intermediate (21-24). If a region of the upstream DNA behind RNAPII interacted with the DNA-peeled part of H2A-H2B, like the foreign DNA observed here, then it would function as a histone transfer intermediate.

The present study unveiled the sequential transitions of nucleosomes during RNAPII passage (Fig. 4). The transcribing RNAPII first encounters the nucleosome [step 1, SHL(6)], and begins its invasion with the peeling of a helical pitch of the DNA segment from the histone surface [step 2, SHL(5)]. The RNAPII elongates the RNA with continuous peeling of the nucleosomal DNA, and stalls at SHL(-2) of the nucleosome (step 3). Finally, RNAPII stalls at SHL(-1) (step 4), where the nucleosome interacts with the foreign DNA (step 5). At or beyond SHL(-1), transcription results in either histone transfer to the upstream DNA behind RNAPII or histone eviction from the DNA. Here, we revealed the structures of the RNAPII-nucleosome complexes formed in the presence of the transcription elongation factor TFIIS. However, in cells, several other elongation factors, including Spt4, Spt5, Spt6, PAF1C, and Elf1, interact with RNAPII $(9,25-27)$, and function in proper transcription in chromatin $(28-30)$. Further studies are needed to understand the mechanism of chromatin transcription by RNAPII.

The nucleosome-dependent transcriptional pause is significant for gene regulation. The first $(+1)$ nucleosome is implicated in the promoter-proximal pausing of transcription, which regulates nucleosomal entry by RNAPII (7). The partially DNA-peeled nucleosome within the paused complexes should be the target of histone chaperones, nucleosome 
remodelers, modification enzymes, etc. Our present RNAPIInucleosome complex structures at the major pause sites shed new light on such regulation mechanisms.

\section{REFERENCES AND NOTES}

1. A. Wolffe, Chromatin: Structure and Function (Academic Press, ed. 3, 1998).

2. K. Luger, A. W. Mäder, R. K. Richmond, D. F. Sargent, T. J. Richmond, Crystal structure of the nucleosome core particle at 2.8 A resolution. Nature $389,251-$ 260 (1997). doi:10.1038/38444 Medline

3. S. S. Teves, C. M. Weber, S. Henikoff, Transcribing through the nucleosome. Trends Biochem. Sci. 39, 577-586 (2014). doi:10.1016/j.tibs.2014.10.004 Medline

4. Y. Lorch, R. D. Kornberg, Chromatin-remodeling for transcription. Q. Rev. Biophys. 50 (e5), e5 (2017). doi:10.1017/S003358351700004X Medline

5. W. K. M. Lai, B. F. Pugh, Understanding nucleosome dynamics and their links to gene expression and DNA replication. Nat. Rev. Mol. Cell Biol. 18, 548-562 (2017). doi:10.1038/nrm.2017.47 Medline

6. P. Cramer, D. A. Bushnell, R. D. Kornberg, Structural basis of transcription: RNA polymerase II at 2.8 angstrom resolution. Science 292, 1863-1876 (2001). doi:10.1126/science. 1059493 Medline

7. C. M. Weber, S. Ramachandran, S. Henikoff, Nucleosomes are context-specific, H2A.Z-modulated barriers to RNA polymerase. Mol. Cell 53, 819-830 (2014). doi:10.1016/j.molcel.2014.02.014 Medline

8. P. T. Lowary, J. Widom, New DNA sequence rules for high affinity binding to histone octamer and sequence-directed nucleosome positioning. J. Mol. Biol. 276, 19-42 (1998). doi:10.1006/jmbi.1997.1494 Medline

9. H. Ehara, T. Yokoyama, H. Shigematsu, S. Yokoyama, M. Shirouzu, S. I. Sekine, Structure of the complete elongation complex of RNA polymerase II with basal factors. Science 357, 921-924 (2017). doi:10.1126/science.aan8552 Medline

10. M. L. Kireeva, B. Hancock, G. H. Cremona, W. Walter, V. M. Studitsky, M. Kashlev, Nature of the nucleosomal barrier to RNA polymerase II. Mol. Cell 18, 97-108 (2005). doi:10.1016/j.molcel.2005.02.027 Medline

11. B. Kastner, N. Fischer, M. M. Golas, B. Sander, P. Dube, D. Boehringer, K. Hartmuth, J. Deckert, F. Hauer, E. Wolf, H. Uchtenhagen, H. Urlaub, F. Herzog, J. M. Peters, D. Poerschke, R. Lührmann, H. Stark, GraFix: Sample preparation for singleparticle electron cryomicroscopy. Nat. Methods 5, 53-55 (2008). doi:10.1038/nmeth1139 Medline

12. F. K. Hsieh, M. Fisher, A. Ujvári, V. M. Studitsky, D. S. Luse, Histone Sin mutations promote nucleosome traversal and histone displacement by RNA polymerase II. EMBO Rep. 11, 705-710 (2010). doi:10.1038/embor.2010.113 Medline

13. B. D. Strahl, C. D. Allis, The language of covalent histone modifications. Nature 403, 41-45 (2000). doi:10.1038/47412 Medline

14. T. Kouzarides, Chromatin modifications and their function. Cell 128, 693-705 (2007). doi:10.1016/j.cell.2007.02.005 Medline

15. A. J. Bannister, T. Kouzarides, Regulation of chromatin by histone modifications. Cell Res. 21, 381-395 (2011). doi:10.1038/cr.2011.22 Medline

16. L. Farnung, S. M. Vos, C. Wigge, P. Cramer, Nucleosome-Chd1 structure and implications for chromatin remodelling. Nature 550, 539-542 (2017). doi:10.1038/nature24046 Medline

17. S. Eustermann, K. Schall, D. Kostrewa, K. Lakomek, M. Strauss, M. Moldt, K. P. Hopfner, Structural basis for ATP-dependent chromatin remodelling by the INO80 complex. Nature 556, 386-390 (2018). doi:10.1038/s41586-018-0029-y Medline

18. R. Ayala, O. Willhoft, R. J. Aramayo, M. Wilkinson, E. A. McCormack, L. Ocloo, D. B. Wigley, X. Zhang, Structure and regulation of the human INO80-nucleosome complex. Nature 556, 391-395 (2018). doi:10.1038/s41586-018-0021-6 Medline

19. Y. Arimura, H. Tachiwana, T. Oda, M. Sato, H. Kurumizaka, Structural analysis of the hexasome, lacking one histone $\mathrm{H} 2 \mathrm{~A} / \mathrm{H} 2 \mathrm{~B}$ dimer from the conventional nucleosome. Biochemistry 51, 3302-3309 (2012). doi:10.1021/bi300129b Medline

20. V. Di Cerbo, F. Mohn, D. P. Ryan, E. Montellier, S. Kacem, P. Tropberger, E. Kallis, M. Holzner, L. Hoerner, A. Feldmann, F. M. Richter, A. J. Bannister, G. Mittler, J. Michaelis, S. Khochbin, R. Feil, D. Schuebeler, T. Owen-Hughes, S. Daujat, R. Schneider, Acetylation of histone $\mathrm{H} 3$ at lysine 64 regulates nucleosome dynamics and facilitates transcription. eLife 3, e01632 (2014). doi:10.7554/eLife.01632 Medline
21. V. M. Studitsky, D. J. Clark, G. Felsenfeld, A histone octamer can step around a transcribing polymerase without leaving the template. Cell 76, 371-382 (1994). doi:10.1016/0092-8674(94)90343-3 Medline

22. J. Bednar, V. M. Studitsky, S. A. Grigoryev, G. Felsenfeld, C. L. Woodcock, The nature of the nucleosomal barrier to transcription: Direct observation of paused intermediates by electron cryomicroscopy. Mol. Cell 4, 377-386 (1999). doi:10.1016/S1097-2765(00)80339-1 Medline

23. C. Hodges, L. Bintu, L. Lubkowska, M. Kashlev, C. Bustamante, Nucleosomal fluctuations govern the transcription dynamics of RNA polymerase II. Science 325, 626-628 (2009). doi:10.1126/science.1172926 Medline

24. L. Bintu, M. Kopaczynska, C. Hodges, L. Lubkowska, M. Kashlev, C. Bustamante, The elongation rate of RNA polymerase determines the fate of transcribed nucleosomes. Nat. Struct. Mol. Biol. 18, 1394-1399 (2011). doi:10.1038/nsmb.2164 Medline

25. C. Bernecky, J. M. Plitzko, P. Cramer, Structure of a transcribing RNA polymerase II-DSIF complex reveals a multidentate DNA-RNA clamp. Nat. Struct. Mol. Biol. 24, 809-815 (2017). doi:10.1038/nsmb.3465 Medline

26. Y. Xu, C. Bernecky, C. T. Lee, K. C. Maier, B. Schwalb, D. Tegunov, J. M. Plitzko, H. Urlaub, P. Cramer, Architecture of the RNA polymerase II-Paf1C-TFIIS transcription elongation complex. Nat. Commun. 8, 15741 (2017). doi:10.1038/ncomms15741 Medline

27. S. M. Vos, L. Farnung, M. Boehning, C. Wigge, A. Linden, H. Urlaub, P. Cramer, Structure of activated transcription complex Pol II-DSIF-PAF-SPT6. Nature 560, 607-612 (2018). doi:10.1038/s41586-018-0440-4 Medline

28. G. A. Hartzog, T. Wada, H. Handa, F. Winston, Evidence that Spt4, Spt5, and Spt6 control transcription elongation by RNA polymerase II in Saccharomyces cerevisiae. Genes Dev. 12, 357-369 (1998). doi:10.1101/gad.12.3.357 Medline

29. D. Prather, N. J. Krogan, A. Emili, J. F. Greenblatt, F. Winston, Identification and characterization of Elf1, a conserved transcription elongation factor in Saccharomyces cerevisiae. Mol. Cell. Biol. 25, 10122-10135 (2005). doi:10.1128/MCB.25.22.10122-10135.2005 Medline

30. J. Kim, M. Guermah, R. G. Roeder, The human PAF1 complex acts in chromatin transcription elongation both independently and cooperatively with SII/TFIIS. Cell 140, 491-503 (2010). doi:10.1016/j.cell.2009.12.050 Medline

31. H. Ehara, T. Umehara, S. I. Sekine, S. Yokoyama, Crystal structure of RNA polymerase II from Komagataella pastoris. Biochem. Biophys. Res. Commun. 487, 230-235 (2017). doi:10.1016/j.bbrc.2017.04.039 Medline

32. C. Bernecky, F. Herzog, W. Baumeister, J. M. Plitzko, P. Cramer, Structure of transcribing mammalian RNA polymerase II. Nature 529, 551-554 (2016). doi:10.1038/nature16482 Medline

33. T. Kujirai, Y. Arimura, R. Fujita, N. Horikoshi, S. Machida, H. Kurumizaka, Methods for preparing nucleosomes containing histone variants. Methods Mol. Biol. 1832, 3-20 (2018). doi:10.1007/978-1-4939-8663-7_1 Medline

34. T. Higo, N. Suka, H. Ehara, M. Wakamori, S. Sato, H. Maeda, S. Sekine, T. Umehara, S. Yokoyama, Development of a hexahistidine-3x FLAG-tandem affinity purification method for endogenous protein complexes in Pichia pastoris. J. Struct. Funct. Genomics 15, 191-199 (2014). doi:10.1007/s10969-014-9190-1 Medline

35. D. N. Mastronarde, Automated electron microscope tomography using robust prediction of specimen movements. J. Struct. Biol. 152, 36-51 (2005). doi:10.1016/j.jsb.2005.07.007 Medline

36. S. Q. Zheng, E. Palovcak, J.-P. Armache, K. A. Verba, Y. Cheng, D. A. Agard, MotionCor2: Anisotropic correction of beam-induced motion for improved cryoelectron microscopy. Nat. Methods 14, 331-332 (2017). doi:10.1038/nmeth.4193 Medline

37. K. Zhang, Gctf: Real-time CTF determination and correction. J. Struct. Biol. 193 1-12 (2016). doi:10.1016/j.jsb.2015.11.003 Medline

38. D. Kimanius, B. O. Forsberg, S. H. Scheres, E. Lindahl, Accelerated cryo-EM structure determination with parallelisation using GPUs in RELION-2. eLife 5, e18722 (2016). doi:10.7554/eLife.18722 Medline

39. D. Vasudevan, E. Y. Chua, C. A. Davey, Crystal structures of nucleosome core particles containing the '601' strong positioning sequence. J. Mol. Biol. 403, 1-10 (2010). doi:10.1016/j.jmb.2010.08.039 Medline

40. P. D. Adams, P. V. Afonine, G. Bunkóczi, V. B. Chen, I. W. Davis, N. Echols, J. J. Headd, L.-W. Hung, G. J. Kapral, R. W. Grosse-Kunstleve, A. J. McCoy, N. W. 
Moriarty, R. Oeffner, R. J. Read, D. C. Richardson, J. S. Richardson, T. C. Terwilliger, P. H. Zwart, PHENIX: A comprehensive Python-based system for macromolecular structure solution. Acta Crystallogr. D 66, 213-221 (2010). doi:10.1107/S0907444909052925 Medline

41. P. Emsley, B. Lohkamp, W. G. Scott, K. Cowtan, Features and development of Coot. Acta Crystallogr. D 66, 486-501 (2010). doi:10.1107/S0907444910007493 Medline

42. A. C. M. Cheung, P. Cramer, Structural basis of RNA polymerase II backtracking, arrest and reactivation. Nature 471, 249-253 (2011). doi:10.1038/nature09785 Medline

43. S. Tagami, S. Sekine, T. Kumarevel, N. Hino, Y. Murayama, S. Kamegamori, M. Yamamoto, K. Sakamoto, S. Yokoyama, Crystal structure of bacterial RNA polymerase bound with a transcription inhibitor protein. Nature 468, 978-982 (2010). doi:10.1038/nature09573 Medline

44. A. Weixlbaumer, K. Leon, R. Landick, S. A. Darst, Structural basis of transcriptional pausing in bacteria. Cell 152, 431-441 (2013). doi:10.1016/i.cell.2012.12.020 Medline

45. S. Sekine, Y. Murayama, V. Svetlov, E. Nudler, S. Yokoyama, The ratcheted and ratchetable structural states of RNA polymerase underlie multiple transcriptional functions. Mol. Cell 57, 408-421 (2015). doi:10.1016/j.molcel.2014.12.014 Medline

46. J. Y. Kang, T. V. Mishanina, M. J. Bellecourt, R. A. Mooney, S. A. Darst, R. Landick, RNA polymerase accommodates a pause RNA hairpin by global conformational rearrangements that prolong pausing. Mol. Cell 69, 802-815.el (2018). doi:10.1016/i.molcel.2018.01.018 Medline

47. E. F. Pettersen, T. D. Goddard, C. C. Huang, G. S. Couch, D. M. Greenblatt, E. C. Meng, T. E. Ferrin, UCSF Chimera-a visualization system for exploratory research and analysis. J. Comput. Chem. 25, 1605-1612 (2004). doi:10.1002/jcc.20084 Medline

\section{ACKNOWLEDGMENTS}

We thank K. Katsura and T. Yokoyama (RIKEN) for their help with the RNAPII preparation and cryo-EM analyses and Y. likura (University of Tokyo) for her assistance. Funding: This work was supported in part by the RIKEN Dynamic Structural Biology project (to M.S., S.S., and H.K.); JSPS KAKENHI grants JP18H05534 (to H.K.), JP25116002 (to H.K.), and JP15H04344 (to S.S.); JST CREST grant JPMJCR16G1 (to H.K.); and the Platform Project for Supporting Drug Discovery and Life Science Research (BINDS) from AMED under grants JP18am0101076 (to H.K.) and JP18am0101082 (to M.S.). Author contributions: T.K. and Y.F. prepared the RNAPII-nucleosome complexes and performed biochemical analyses. H.E., T.K., M.S., and S.S. performed cryo-EM analyses. S.S. and H.K. conceived, designed, and supervised all of the work. T.K., H.E., S.S., and H.K. wrote the paper. All of the authors discussed the results and commented on the manuscript. Competing interests: The authors declare no competing interests. Data and materials availability: The cryo-EM reconstructions and atomic models of the RNAPII-nucleosome complexes have been deposited in the Electron Microscopy Data Bank and the Protein Data Bank (PDB) under the following accession codes: EMD-6981 and PDB ID 6 A50 for RNAPII complex stalled at SHL(-6) of the nucleosome; EMD-6982 and PDB ID 6A5P for RNAPII complex stalled at SHL(-5) of the nucleosome; EMD-6983 and PDB ID 6A5P for RNAPII complex stalled at SHL(-2) of the nucleosome; EMD6984 and PDB ID 6A5T for RNAPII complex stalled at SHL(-1) of the nucleosome; EMD-6980 and PDB ID 6A5L for RNAPII complex stalled at SHL(-1) of the nucleosome, with foreign DNA; EMD-6985 and PDB ID 6A5U for RNAPII complex stalled at SHL(-1) of the nucleosome, with foreign DNA, tilt conformation; EMD6986 and PDB ID 6A5V for RNAPII complex stalled at SHL(-1) +1 of the nucleosome, with foreign DNA.

\section{SUPPLEMENTARY MATERIALS}

www.sciencemag.org/cgi/content/full/science.aau9904/DC1 Materials and Methods

Figs. S1 to S11

Tables S1 and S2

References (33-47)

Movies S1 and S2

3 August 2018; accepted 19 September 2018

Published online 4 October 2018

10.1126/science.aau9904 


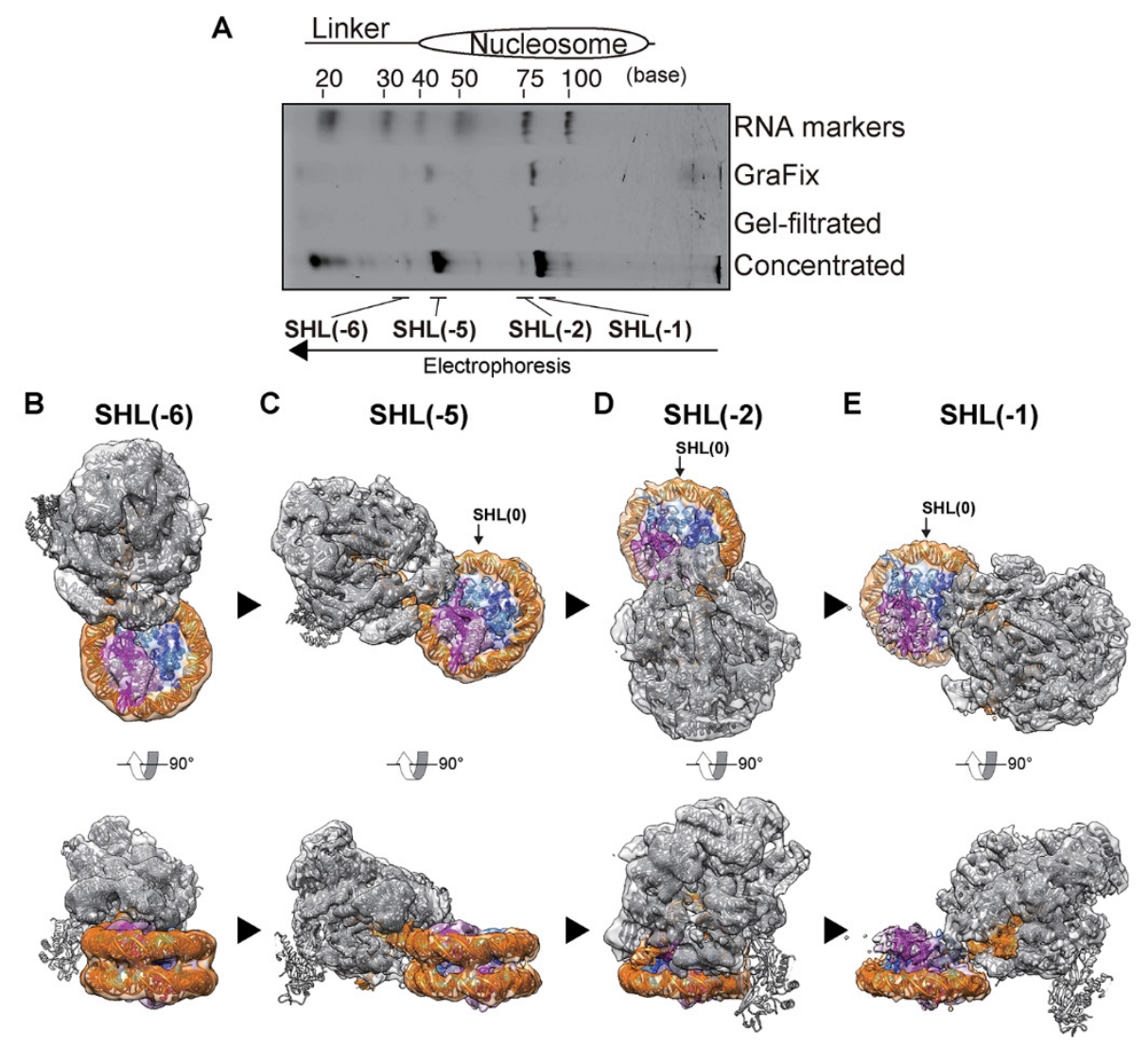

Fig. 1. Cryo-EM structures of the transcribing RNAPII-nucleosome complexes. (A) Elongated RNAs in the purified transcribing RNAPIInucleosome complexes were analyzed by denaturing gel electrophoresis. After the transcription reaction, the complexes were fractionated by the GraFix method (lane: GraFix), desalted on a gel filtration column (lane: Gel-filtrated), and concentrated (lane: Concentrated). (B to E) Cryo-EM densities of the RNAPII-nucleosome complexes paused at SHL(-6) (B), SHL(-5) (C), SHL(-2) (D), and SHL(-1) (E) with fitted structural models. The density for the Rpb4/7 stalk of RNAPII is weak, because of its inherent flexibility $(31,32)$. Focused classification clearly reveals the stalk density (fig. S11). RNAPII, DNA, $\mathrm{H} 2 \mathrm{~A}, \mathrm{H} 2 \mathrm{~B}, \mathrm{H} 3$, and $\mathrm{H} 4$ are colored gray, orange, magenta, pink, light blue, and blue, respectively. The arrows indicate the $\mathrm{SHL}(0)$ positions. 


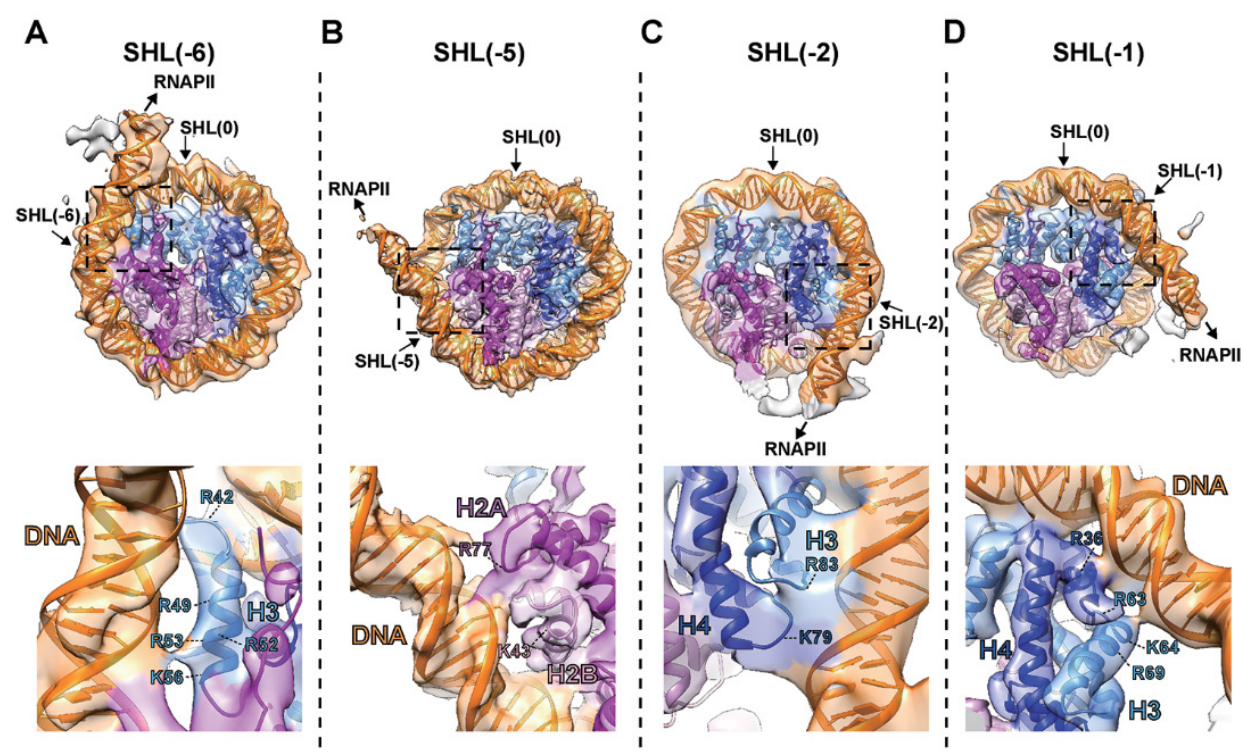

Fig. 2. Structural transition of the nucleosome. Improved density maps after focused refinement (see materials and methods) for the SHL(-6) (A), SHL(-5) (B), SHL(-2) (C), and SHL(-1) (D) complexes are shown with the atomic models. Top panels indicate overall nucleosome structures. These views are the same orientations as those in Fig. 1, B to E (upper panels). Close-up views of the regions encircled by the dashed rectangles in the top panels are presented in the bottom panels. The arginine and lysine residues that potentially contact the DNA at the RNAPII pausing sites are highlighted. 
A

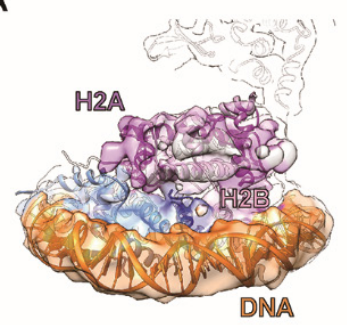

B

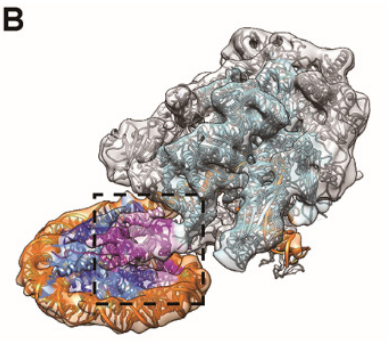

C

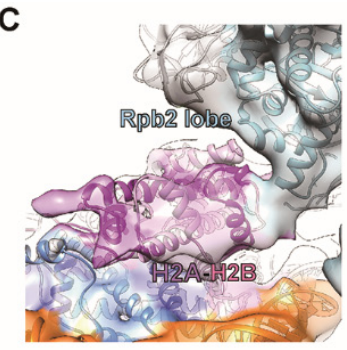

Fig. 3. Interaction between an $\mathrm{H} 2 \mathrm{~A}-\mathrm{H} 2 \mathrm{~B}$ dimer and RNAPII. (A) A close-up view of an $\mathrm{H} 2 \mathrm{~A}-\mathrm{H} 2 \mathrm{~B}$ dimer in the nucleosomes of the $\mathrm{SHL}(-1)$ complex. Cryo-EM density maps of the nucleosomes in the SHL(-1) complex, fitted with the structure. (B) The overall density map with the atomic model structure of the $\mathrm{SHL}(-2)$ complex. Rpb2 of RNAPII is colored pale blue. (C) The region surrounded by a dashed square in (B) is enlarged. 


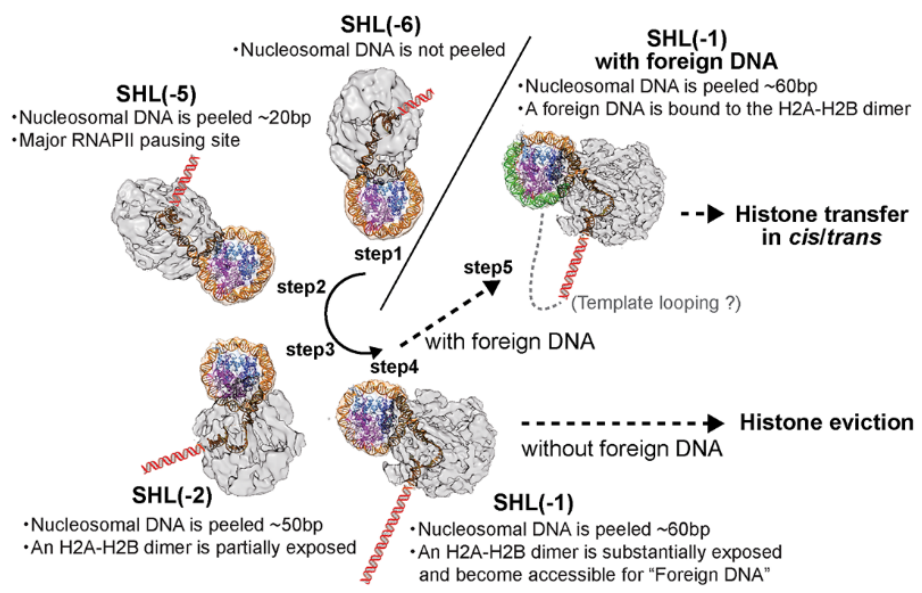

Fig. 4. Scheme of transcription elongation through a nucleosome by RNAPII. RNAPII encounters a nucleosome [SHL(-6); step 1], and then proceeds to SHL(-5), which is a major pausing site for RNAPII (step 2). After passing through SHL(-5), the RNAPII pauses $\mathrm{SHL}(-2)$ (step 3), and proceeds to SHL(-1) (step 4). In the $\mathrm{SHL}(-2)$ and $\mathrm{SHL}(-1)$ complexes, an $\mathrm{H} 2 \mathrm{~A}-\mathrm{H} 2 \mathrm{~B}$ dimer is exposed. Then, foreign DNA is bound to the DNA-peeled region of the nucleosome in the complex (step 5). Histones may be removed from the region ahead of the RNAPII, by transfer in cis/trans or eviction. The red DNA cartoons indicate possible orientations of the upstream DNAs. 